\title{
Atypical tuberculosis of the knee joint
}

\section{G Albuquerque-Jonathan, MB ChB, FCRad (D) \\ Department of Radiology, Groote Schuur Hospital, Cape Town}

This case report demonstrates the MRI findings of atypical tuberculosis (TB) of the knee joint, caused by Mycobacterium kansasii. Osteoarticular TB caused by atypical mycobacteria is rare; instead, it is predominantly a synovial disease affecting the tendon sheaths rather than bone. Predisposing factors are immunocompromised individuals, including the elderly, alcoholics, those with HIV, diabetes mellitus, malnutrition and other chronic debilitating illnesses, and local factors, such as preexisting trauma, rheumatoid arthritis, systemic lupus erythematosis, and prosthetic joints. We present a case that occurred in an individual without any predisposing factors, which is also a rare occurrence.

\section{Case report}

A 26-year-old woman presented with a 6-year history of knee pain and swelling. Her daily activities were affected, and the pain also kept her up at night. There was some response to analgesics. She had no history of trauma, and the onset has been insidious. No other joints were affected. There was a background of possible pulmonary TB, but treatment for this had been started and subsequently stopped. Some weight loss had occurred.

Clinically she had a monoarthritis with evidence of synovitis and an effusion. Laboratory tests revealed a raised erythrocyte sedimentation rate and C-reactive protein. Rheumatoid factor was negative. Plain radiographs revealed peri-articular osteopaenia and a joint effusion.

An MRI was performed to further evaluate these changes (Fig. 1). The MRI showed extensive synovitis, with bone marrow oedema and erosions. The erosions involved both bone and meniscal margins. Multiple lymph nodes were noted in the popliteal fossa. The overall appearance is non-specific, with the differential diagnosis including all inflammatory arthritides, such as rheumatoid arthritis, pigmented villonodular synovitis and TB.

The patient underwent both synovial fluid aspiration and biopsy. The joint fluid was serous, with lymphocytes present, but there was no culture growth after 6 weeks. The synovial biopsy was normal on microscopy and Ziehl-Nielsen stain, but after 24 hours of incubation for acid-fast bacilli, Mycobacterium kansasii was cultured. The patient was placed on TB treatment for 12 months and is presently still being followed up.

\section{Radiological findings of TB arthritis}

Plain radiographs only demonstrate changes after 3 - 4 weeks. Early findings are a joint effusion and soft tissue swelling. Later findings encompass the classic 'Phemister triad', which comprises joint space narrowing, juxta-articular osteopaenia and erosions. An important feature of TB of the joints can be seen in superficial joints, e.g. hands, where reactive subperiosteal new bone formation appears around lytic areas. ${ }^{1}$

MRI of TB arthritis can present with a wide range of findings. ${ }^{2}$ These include bone marrow oedema, synovitis, cartilage/bony destruction, myositis, cellulitis, granulation tissue, abscess-fistula, tenosynovitis, bursitis, fibrosis, etc. ${ }^{2}$

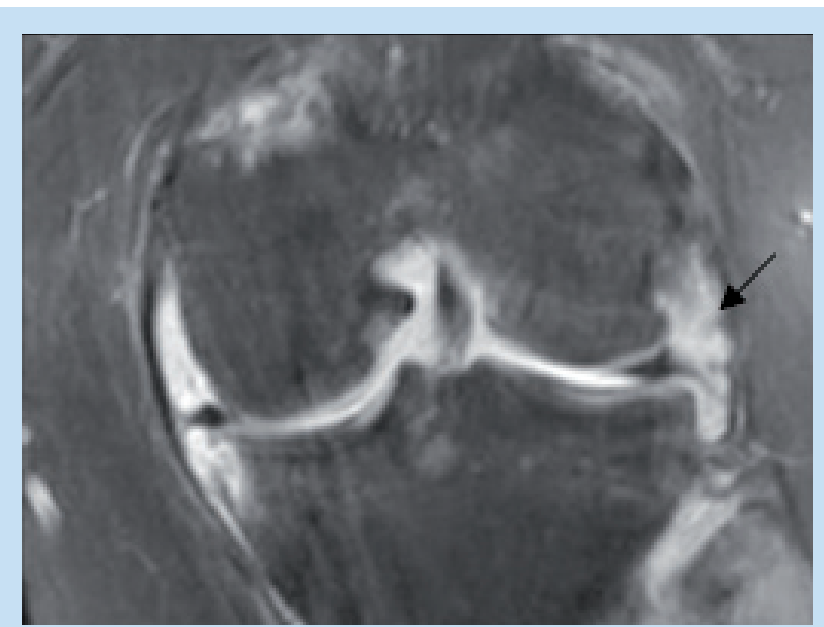

Fig. 1. Proton density fat-saturated MRI. Coronal image demonstrating marginal bone and cartilage erosions, synovial proliferation (arrow) and joint effusion.

Findings which should alert one to the possibility of TB include T1WI hyperintense soft tissue, which may represent caseous material, and a T1WI hyperintense lining of a bursitis, a smooth, thin enhancing bursal margin, and bone destruction with new bone formation.

Atypical TB of joints is unusual and when it does occur, it involves synovial sheaths more than bones. Often there are underlying predisposing conditions, most commonly, HIV or AIDS. Mycobacterium kansasii is the commonest atypical organism found in osteoarticular TB. Others reported include $M$. xenopi and M. avium intracellulare.

The diagnosis is often not made on clinical and imaging grounds. Any patient with a persistent monoarthritis which does not respond to intra-articular steroid injection, requires a synovial biopsy for definitive diagnosis. Adequate synovial biopsy has a yield of $90 \%$.

Primary management is anti-TB medication. ${ }^{1}$ Surgical management is occasionally indicated where there has been an unsatisfactory response to drug treatment, extensive bone involvement, or cold abscess formation, when synovectomy, drainage or debridement may be required. In later stages, where there is residual joint deformity, instability or subluxation, splinting, osteotomy or arthroplasty may be indicated. Partial synovectomy and more complex surgical procedures are restricted to joints that demonstrate severe cartilage destruction, joint deformity, abscess formation, multi-drug resistance or atypical mycobacteria.

\section{Conclusion}

Atypical TB of the joints is a rare finding and a high index of suspicion is required to make the diagnosis. Radiological features are often non-specific, and histopathology is required to confirm the diagnosis. Radiological features that may alert one to the presence of TB, include T1WI hyperintensity within the joint and bursal margins, representing caseation.

\footnotetext{
1. Malaviya AN, Kotwal PP. Arthritis associated with tuberculosis. Best Pract Res Clin Rheumatol 2003; 17: 319-343.

2. Parmar H, Shah J, Patkar D, Singrakhia M, Patankar T, Hutchinson C. Tuberculous arthritis of the appendicular skeleton: MR imaging appearances. Eur J Radiol 2004; 52: 300-309.
} 